\title{
PERFIL SOCIODEMOGRÁFICO DE GESTANTES EN EL PERÚ SEGÚN REGIONES NATURALES
}

\author{
Marcos Espinola-Sánchez ${ }^{1, a}$, Augusto Racchumí-Vela ${ }^{2, b}$, Pedro Arango-Ochante ${ }^{3, c}$, Percy Minaya-León $^{3, a}$
}

\begin{abstract}
RESUMEN
Objetivos. Describir el perfil sociodemográfico de gestantes en el Perú según regiones naturales. Materiales y métodos. Estudio realizado a partir de la fuente de datos de ENDES 2014-2018, se incluyeron 5464 gestantes que cumplieron criterios de selección. Se aplicó estadística descriptiva y estadística inferencial. Resultados. Embarazo adolescente en la Costa fue de 13\%, embarazo en añosas 15.3\%; en la región de la Sierra el embarazo adolescente $14.7 \%$ y el embarazo en añosas $16.9 \%$; en la región de la Selva el embarazo adolescente fue del $17.1 \%$ y el embarazo en añosas del $13.4 \%$. Las mujeres gestantes que refirieron que no tenían la preferencia de quedar embarazadas en ese momento actual, fue en la Costa $62.4 \%$, Sierra $62.1 \%$ y en la Selva $59.4 \%$. Las gestantes que proceden de la Costa con mayor frecuencia tienen estudios de secundaria y pertenecer a un quintil intermedio de riqueza. Las gestantes que procedían de la Selva o la Sierra presentaron con mayor frecuencia estudios de solo primaria y pertenecer al quintil inferior de riqueza. Conclusiones. Existen diferencias en el perfil sociodemográfico de mujeres gestantes según regiones naturales en nuestro país, donde la edad, el nivel educativo, quintil de riqueza, deseos de quedar embarazada representan un papel importante que contribuyen a conocer la evolución y las tendencias en el perfil de las gestantes.
\end{abstract}

Palabras Clave. Embarazo; Práctica sexual (Fuente: DeCS BIREME).

\section{SOCIODEMOGRAPHIC PROFILE OF GESTANTES IN PERU ACCORDING TO NATURAL REGIONS}

\begin{abstract}
Objectives. Describe the sociodemographic profile of pregnant women in Peru according to natural regions. Materials and methods. This study was conducted from the data source of ENDES 2014-2018, included 5464 pregnant women who met the selection criteria. Descriptive statistics and inferential statistics were applied. Results. In the Costa region, adolescent pregnancy represented $13 \%$ and pregnancy in the elderly was $15.3 \%$; in the Sierra region, adolescent pregnancy represented $14.7 \%$ and pregnancy in the elderly was $16.9 \%$; while, in the Selva region, adolescent pregnancy was $17.1 \%$ and pregnancy in years $13.4 \%$. The pregnant women mainly reported that they did not have the preference of becoming pregnant at that time, being $62.4 \%$ more frequently in the Costa region, followed by the Sierra $62.1 \%$ and in the Jungle $59.4 \%$. Pregnant women who come from the Coast most often have only secondary studies and belong to an intermediate wealth quintile. The pregnant women who came from the Selva or the Sierra presented more often studies of only primary and belong to the lower wealth quintile. Conclusions. There are differences in the sociodemographic profile of pregnant women according to natural regions in our country, age, educational level, wealth quintile, desires to become pregnant represent an important role that contribute to knowing the evolution and trends in the profile of pregnant women.
\end{abstract}

Keywords: Pregnancy; Sexual practice (Source: MeSH NLM).

\section{INTRODUCCIÓN}

La salud materna es uno de los objetivos esenciales en el desarrollo de los países ${ }^{1,2}$, si bien existen indicadores para su monitorización como la mortalidad materna y la morbilidad materna extrema ${ }^{3,4}$, es solo el desenlace final, haciéndose necesario conocer el contexto del embarazo en cada población para adecuar la mejora en las decisiones de salud pública ${ }^{1,4}$. La salud materna resulta de la interacción variada de múltiples condiciones no solo de tipo biológico sino también de acceso a los servicios de salud, factores sociales, económicos y educativos que contribuyen a la aparición de complicaciones que podrían ser evitadas ${ }^{1,2}$; de igual modo, contribuye el desarrollo de tamizajes de detección oportuna como VIH y sífilis en la gestación ${ }^{5,6}$.

Las desigualdades sociales están relacionadas con la salud, a medida que desciende el nivel socioeconómico los indicadores de salud muestran peores resultados en la mortalidad, la utilización de servicios preventivos, y el acceso a servicios sanitarios ${ }^{7,8}$. Así mismo, el embarazo

Universidad Alcalá de Henares. Madrid-España.

Universidad Nacional Pedro Ruiz Gallo. Chiclayo-Perú.

Universidad Nacional Mayor de San Marcos. Lima-Perú.

a Médico cirujano.

b Licenciado en Estadística.

Médico Gineco-obstetra.

Citar como: Espinola-Sanchez M, Racchumi-Vela A, Arango-Ochante P, Minaya-León P. Perfil Sociodemográfico de gestantes en el Perú según regiones naturales. Rev Peru Investig Matern Perinat 2019; 8(2):14-20.

DOI: https://doi.org/10.33421/inmp.2019149

Recibido: 15-05-19 Aprobado: 28-06-19 
es una condición que no solo limita laboralmente las oportunidades en el entorno organizacional9,10,11; sino que también, las mujeres se enfrentan al reto de conciliar los roles maternal y profesional conllevando a la afectación del estado psicosocial; y puede ser nocivo para la salud y la seguridad de las gestantes ${ }^{12,13}$.

Por otro lado, la maternidad entendida como producto sociocultural ha ido modificándose en las últimas décadas, la mayor autonomía económica, la anticoncepción, el valor del tiempo libre y la mayor formación educativa, entre otros factores, hacen que la maternidad ya no represente un fin para las mujeres, sino una posibilidad más entre otras en la planificación de su vida ${ }^{14,15}$. Estas modificaciones del perfil sociocultural influyen en las diversas formas de actuar de las mujeres entorno a la maternidad, en la cual también influyen aspectos relacionados a su edad y condiciones de vida ${ }^{15,16}$.

\section{MATERIALES Y MÉTODOS}

Tipo de estudio. Estudio descriptivo multivariado, a partir de la base de datos de La Encuesta Demográfica y de Salud Familiar (ENDES). Área de estudio. Los datos de la ENDES proceden de mujeres a nivel nacional entre 2014 al 2018, obtenido mediante un muestreo bietápico, probabilístico e independiente a nivel departamental y por área Urbana y Rural. Población y muestra. Durante el periodo de estudio la ENDES reporto 161937 mujeres encuestadas, de las cuales 5918 se encontraban embarazadas, y de ellas 5464 embarazadas cumplieron con los siguientes criterios de selección: gestantes con registro de las variables socioculturales de interés y no pérdida de datos. Variables. Se recogieron edad clasificándolo según grupo etario en gestantes adolescentes (10-19 años), gestantes adultas (20-34 años) y gestantes añosas (de 35 años a más), nivel educativo, quintil de riqueza, preferencia de quedar embarazada, lugar de residencia, empleo, región natural. Técnicas e instrumentos. Se realizó una integración de las bases de datos de ENDES 2014 al 2018, recodificación de las variables de interés y su extracción a la data en el programa SPSS y el programa $\mathrm{R}$ para limpieza de datos perdidos. Procedimientos. Las bases de datos fueron obtenidas desde los microdatos de la base de datos en la página oficial del Instituto Nacional de Estadística e Informática del Perú. Las bases de datos de los periodos 2014-2018 fueron integradas mediante la identificación de llaves primarias como indicador de cada gestante encuestada, la recodificación de las variables se realizó mediante sintaxis condicionales en el programa SPSS. La limpieza de la data para evitar datos perdidos fue realizada en el programa estadístico R. Aspectos éticos. Debido a la naturaleza del tipo de estudio no se contó con la identidad de las participantes, ni se trabajó directamente con ellas; sino con una fuente de datos primarios de acceso libre y de forma gratuita. Análisis de datos. Se utilizó estadística descriptiva mediante distribución de frecuencias y porcentajes y estadística inferencial con un $p<0,05$ para comparar entre regiones naturales según variables de interés. Se realizó el análisis multivariado de correspondencia para visualizar las relaciones de las categorías de las variables del estudio. Los datos fueron procesados en los programas estadísticos SPSS versión 25 y el programa R versión 3.6.

\section{RESULTADOS}

En la región de la Costa el embarazo adolescente represento el $13 \%$ y el embarazo en añosas fue del $15.3 \%$; en la región de la Sierra el embarazo adolescente represento el $14.7 \%$ y el embarazo en añosas fue del $16.9 \%$; mientras que, en la región de la Selva el embarazo adolescente fue del $17.1 \%$ y el embarazo en añosas del 13.4\%. (Tabla 1).

El lugar de residencia de gestantes en la Costa es con mayor frecuencia urbano $(90.2 \%)$, seguido de rural $(9.8 \%)$; en la Sierra es rural $(55.7 \%)$ seguido de urbano (44.3\%); mientras que en la selva es urbano $(53.5 \%)$ seguido de rural $(46.5 \%)$ (Tabla 1$)$.

Las gestantes de la región Sierra y Selva se encontraron con mayor frecuencia en quintiles inferiores de riqueza; mientras que, en la Costa varío encontrándose principalmente gestantes en el quintil intermedio (28.5\%), seguido del segundo quintil (24.6\%) y en menor frecuencia en el quintil inferior (6.5\%). Según nivel de educación las gestantes con estudios primarios se encontraron principalmente en la región de la Selva $32.5 \%$, seguido de la Sierra $26.6 \%$ y la Costa con el $10.6 \%$ (Tabla 1 ).

Las mujeres gestantes refirieron principalmente que no tenían la preferencia de quedar embarazadas en ese momento actual, siendo con mayor frecuencia en la región de la Costa $62.4 \%$, seguida de la Sierra $62.1 \%$ y en la Selva el $59.4 \%$ (Tabla 1 ).

El embarazo adolescente es más frecuente entre embarazadas de la región Selva en comparación con la región de la Costa $(p<0.05)$, sin embargo no existen diferencias significativas entre la frecuencia de embarazo adolescente entre la región de la Selva y la Sierra $(p>0.05)$. El lugar de residencia rural de las embarazadas en la región Sierra es más frecuente en comparación que en la Costa y en la Selva $(p<0.05)$. El quintil inferior de riqueza es más frecuente en gestantes de la Sierra y la selva en comparación con las gestantes de la región Costa de forma significativa $(p<0.05)$. El nivel de educación primaria fue más frecuente significativamente en la Selva en comparación con la Costa y la Sierra $(p<0.05)$ (tabla 2).

En la Costa la tendencia anual del embarazo adolescente varía, presentando una disminución del $16 \%$ al $12.7 \%$, en la Sierra varía del $15.3 \%$ al $13.7 \%$; sin embargo en la región de la Selva se evidencio un incremento del embarazo adolescente entre las gestantes de esta región, variando entre el $16 \%$ al $19 \%$ (figura 1 ). 
Tabla 1. Características sociodemográficas de gestantes en el Perú. ENDES 2014 al 2018

\begin{tabular}{|c|c|c|c|c|c|c|c|c|c|c|}
\hline \multirow{3}{*}{\multicolumn{2}{|c|}{ Características de la gestante }} & \multicolumn{7}{|c|}{ Región Natural } & \multirow{2}{*}{\multicolumn{2}{|c|}{$\begin{array}{c}\text { Prueba de } \\
\text { independencia }\end{array}$}} \\
\hline & & \multicolumn{2}{|c|}{ Costa } & \multicolumn{2}{|c|}{ Sierra } & \multicolumn{2}{|c|}{ Selva } & \multirow[b]{2}{*}{$\%$} & & \\
\hline & & $\mathbf{N}$ & $\mathbf{n}$ & $\%$ & $\mathbf{n}$ & $\%$ & $\mathbf{n}$ & & Chi2 & Sig \\
\hline \multirow{4}{*}{ Grupo de edad } & 10 a 19 años & 804 & 293 & $13.0 \%$ & 238 & $14.7 \%$ & 273 & $17.1 \%$ & \multirow{4}{*}{18.67} & \multirow{4}{*}{, $001^{*}$} \\
\hline & 20 a 34 años & 3827 & 1610 & $71.7 \%$ & 1110 & $68.4 \%$ & 1107 & $69.4 \%$ & & \\
\hline & 35 a más & 833 & 344 & $15.3 \%$ & 275 & $16.9 \%$ & 214 & $13.4 \%$ & & \\
\hline & Subtotal & 5464 & 2247 & $100.0 \%$ & 1623 & $100.0 \%$ & 1594 & $100.0 \%$ & & \\
\hline \multirow{3}{*}{$\begin{array}{l}\text { Lugar de } \\
\text { residencia }\end{array}$} & Rural & 1866 & 220 & $9.8 \%$ & 904 & $55.7 \%$ & 742 & $46.5 \%$ & \multirow{3}{*}{1037.02} & \multirow{3}{*}{, $000^{*}$} \\
\hline & Urbano & 3598 & 2027 & $90.2 \%$ & 719 & $44.3 \%$ & 852 & $53.5 \%$ & & \\
\hline & Subtotal & 5464 & 2247 & $100.0 \%$ & 1623 & $100.0 \%$ & 1594 & $100.0 \%$ & & \\
\hline \multirow{6}{*}{$\begin{array}{l}\text { Quintil de } \\
\text { riqueza }\end{array}$} & Quintil inferior & 1675 & 147 & $6.5 \%$ & 753 & $46.4 \%$ & 775 & $48.6 \%$ & \multirow{6}{*}{1447.00} & \multirow{6}{*}{, $000^{*}$} \\
\hline & Segundo quintil & 1494 & 552 & $24.6 \%$ & 455 & $28.0 \%$ & 487 & $30.6 \%$ & & \\
\hline & Quintil intermedio & 1052 & 641 & $28.5 \%$ & 219 & $13.5 \%$ & 192 & $12.0 \%$ & & \\
\hline & Cuarto quintil & 766 & 529 & $23.5 \%$ & 130 & $8.0 \%$ & 107 & $6.7 \%$ & & \\
\hline & Quintil superior & 477 & 378 & $16.8 \%$ & 66 & $4.1 \%$ & 33 & $2.1 \%$ & & \\
\hline & Subtotal & 5464 & 2247 & $100.0 \%$ & 1623 & $100.0 \%$ & 1594 & $100.0 \%$ & & \\
\hline \multirow{5}{*}{$\begin{array}{l}\text { Nivel de } \\
\text { educación }\end{array}$} & Sin nivel & 97 & 13 & $0.6 \%$ & 50 & $3.1 \%$ & 34 & $2.1 \%$ & \multirow{5}{*}{420.94} & \multirow{5}{*}{, $000^{*}$} \\
\hline & Primaria & 1188 & 238 & $10.6 \%$ & 432 & $26.6 \%$ & 518 & $32.5 \%$ & & \\
\hline & Secundaria & 2653 & 1141 & $50.8 \%$ & 753 & $46.4 \%$ & 759 & $47.6 \%$ & & \\
\hline & Superior & 1526 & 855 & $38.1 \%$ & 388 & $23.9 \%$ & 283 & $17.8 \%$ & & \\
\hline & Subtotal & 5464 & 2247 & $100.0 \%$ & 1623 & $100.0 \%$ & 1594 & $100.0 \%$ & & \\
\hline \multirow{3}{*}{$\begin{array}{l}\text { Preferencia } \\
\text { de quedar } \\
\text { embarazada }\end{array}$} & En ese momento & 2109 & 846 & $37.7 \%$ & 616 & $38.0 \%$ & 647 & $40.6 \%$ & \multirow{3}{*}{3.803} & \multirow{3}{*}{0.149} \\
\hline & $\begin{array}{l}\text { Después o no quería } \\
\text { en ese momento }\end{array}$ & 3355 & 1401 & $62.4 \%$ & 1007 & $62.1 \%$ & 947 & $59.4 \%$ & & \\
\hline & Subtotal & 5464 & 2247 & $100.0 \%$ & 1623 & $100.0 \%$ & 1594 & $100.0 \%$ & & \\
\hline
\end{tabular}



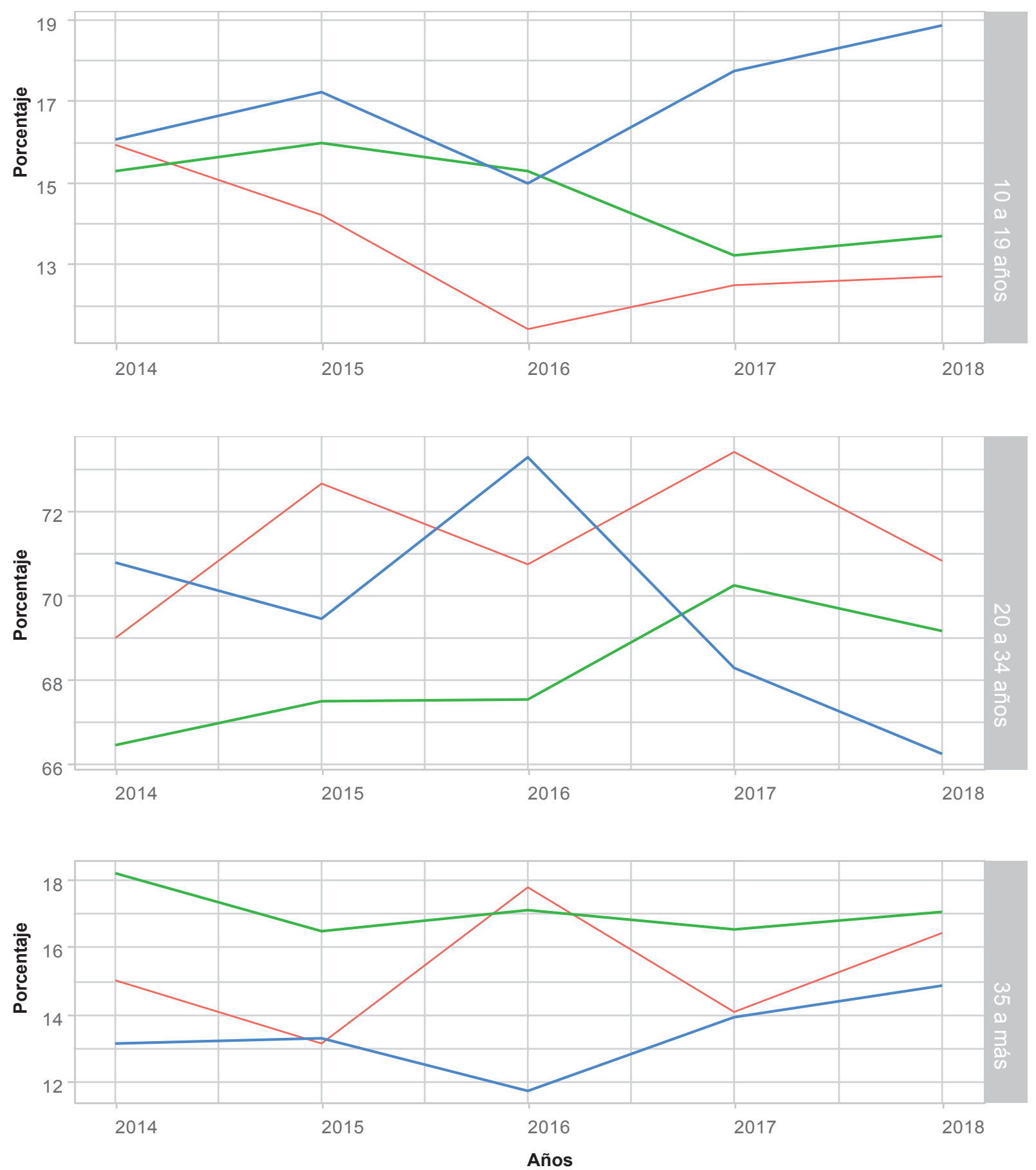

Región Natural - Costa - Sierra - Selva

Figura 1. Tendencia anual de gestantes según grupo etario y region natural en el Perú. ENDES 2014 al 2018 
Tabla 2. Diferencias significativas de características sociodemográficas según región natural. ENDES 2014 al 2018

\begin{tabular}{|c|c|c|c|c|}
\hline \multicolumn{2}{|c|}{ Características de las gestantes } & \multicolumn{3}{|c|}{ Región Natural } \\
\hline & & $\begin{array}{c}\text { Costa } \\
\text { (A) }\end{array}$ & $\begin{array}{c}\text { Sierra } \\
(B)\end{array}$ & $\begin{array}{c}\text { Selva } \\
\text { (C) }\end{array}$ \\
\hline \multirow{3}{*}{$\begin{array}{l}\text { Grupo } \\
\text { de edad }\end{array}$} & 10 - 19 ค ว & & & $A$ \\
\hline & 20 a 34 años & & & \\
\hline & 35 a más & & C & \\
\hline \multirow{2}{*}{$\begin{array}{l}\text { Lugar de } \\
\text { residencia }\end{array}$} & Rural & & $\begin{array}{l}A \\
C\end{array}$ & $A$ \\
\hline & Urbano & $\begin{array}{l}\mathrm{B} \\
\mathrm{C}\end{array}$ & & B \\
\hline \multirow{5}{*}{$\begin{array}{l}\text { Quintil de } \\
\text { riqueza }\end{array}$} & Quintil inferior & & $A$ & A \\
\hline & Segundo quintil & & A & A \\
\hline & Quintil intermedio & $\begin{array}{l}\mathrm{B} \\
\mathrm{C}\end{array}$ & & \\
\hline & Cuarto quintil & $\begin{array}{l}\mathrm{B} \\
\mathrm{C}\end{array}$ & & \\
\hline & Quintil superior & $\begin{array}{l}\mathrm{B} \\
\mathrm{C}\end{array}$ & C & \\
\hline \multirow[t]{2}{*}{ Empleo } & Sin empleo & $\begin{array}{l}\mathrm{B} \\
\mathrm{C}\end{array}$ & & \\
\hline & Empleada & & $A$ & $A$ \\
\hline \multirow{4}{*}{$\begin{array}{l}\text { Nivel de } \\
\text { Educación }\end{array}$} & Sin nivel & & A & A \\
\hline & Primaria & & A & $\begin{array}{l}A \\
B\end{array}$ \\
\hline & Secundaria & B & & \\
\hline & Superior & $\begin{array}{l}\mathrm{B} \\
\mathrm{C}\end{array}$ & C & \\
\hline
\end{tabular}

Se evidenció una relación múltiple entre gestantes que procede de la Costa, tener una edad de 20 a 34 años y haber preferido estar embarazadas en ese momento actual, así como entre tener estudios de solo secundaria y pertenecer a un quintil intermedio de riqueza; mientras que, las gestantes que presentaron educación superior se encontraban en el cuarto quintil de riqueza. Por otro lado, las gestantes que procedían de la Selva o la Sierra se relacionaron a tener estudios de solo primaria y pertenecer al quintil inferior de riqueza. Las gestantes adolescentes que en ese momento no querían estar embarazadas estuvo asociado a las que procedían de la región Costa en comparación de la región Sierra y Selva (figura 2).

\section{DISCUSIÓN}

La salud materna tiene como resultado la sumatoria de factores económicos, educacionales, nutricionales y de acceso a redes de protección social en un país 1 ; así mismo, en las últimas décadas la representación de la mujer como un binomio, el de mujer igual madre; ha presentado modificaciones en la construcción sociocultural de la maternidad y por ende modificaciones en la percepción del embarazo14.

En el Perú, el embarazo principalmente corresponde a mujeres en edades de 20 a 34 años en las tres regiones naturales; sin embargo, existe un alto índice de embarazo adolescente en la región Selva de $17.1 \%$, en la costa de $14.7 \%$ y en la Costa de $13 \%$ de las embarazadas, mientras que en las regiones Costa y Sierra se encuentra
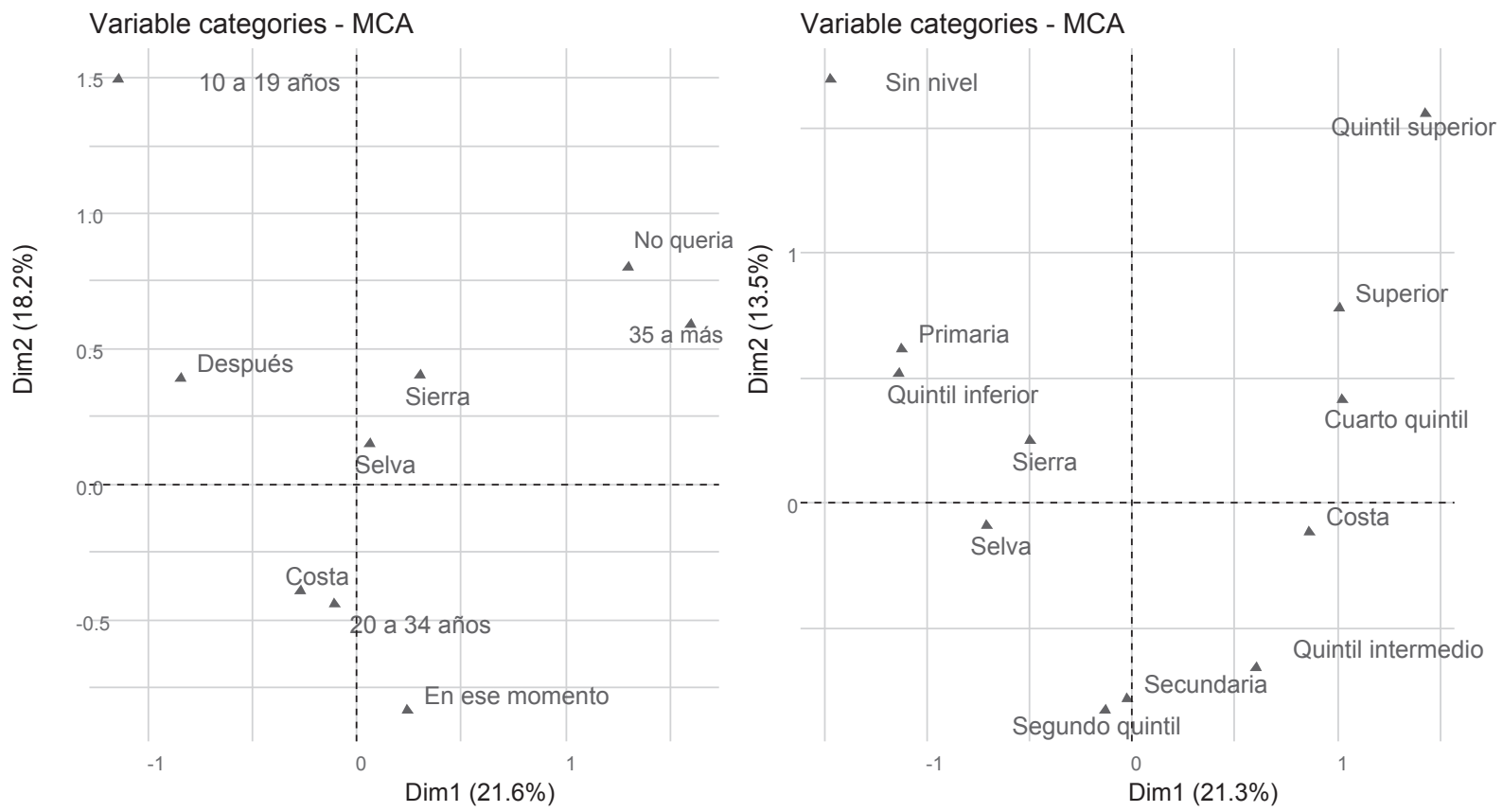

Figura 2. Correlación entre características sociodemográficas de gestantes en el Perú. ENDES 2014 al 2018 
en segundo lugar el embarazo en gestantes añosas con el $15.3 \%$ y $16.9 \%$ respectivamente. De igual forma, reportes previos evidencian una persistente heterogeneidad en el embarazo adolescente, que oscila entre el 12 y $13 \%$, sobre todo si no es planificado, que como se observa es la mayoría de casos18. Cabe resaltar en reportes previos del embarazo adolescente que al compararlo con los hombres, menos de $1 \%$ de ellos son padres a la misma edad18; así mismo, en diferentes estudios existen diferentes factores que influyen en las modificaciones de la maternidad como

En la presente investigación se evidenció una relación múltiple entre gestantes que procede de la Costa, tener una edad de 20 a 34 años y haber preferido estar embarazadas en ese momento actual, así como tener estudios de solo secundaria y pertenecer a un quintil intermedio de riqueza. Sin embargo, las gestantes que procedían de la Selva o la Sierra se relacionaron a tener estudios de solo primaria y pertenecer al quintil inferior de riqueza.

Aunque las prevalencias de embarazo adolescente son menores en áreas urbanas, es aquí donde se registra una leve tendencia al aumento, a diferencia de la tendencia al descenso registrada en las áreas rurales, pero persistentemente en áreas de la selva ${ }^{19,20}$.

Las gestantes adolescentes que en ese momento no querían estar embarazadas estuvo asociado a las que procedían de la región Costa en comparación de la región Sierra y Selva. De forma similar a otros estudios se evidencia que cada vez son más las adolescentes embarazadas que hubieran querido ese embarazo más tarde; mientras, que las cifras de las que efectivamente buscaban quedar embarazadas han descendido ${ }^{21}$. Se trata de situaciones que responden a cambios en la mentalidad, en los planes y expectativas de vida, y a una mayor educación y acceso a información en múltiples medios $^{16}$.

Las gestantes de la región Sierra y Selva se encontraron con mayor frecuencia en quintiles inferiores de riqueza; mientras que, en la Costa varío encontrándose principalmente gestantes en el quintil intermedio (28.5\%), seguido del segundo quintil (24.6\%) y en menor frecuencia en el quintil inferior (6.5\%). Según nivel de educación las gestantes con estudios primarios se encontraron principalmente en la región de la Selva $32.5 \%$, seguido de la Sierra $26.6 \%$ y la Costa con el $10.6 \%$. Esto es similar a otras publicaciones donde los estratos de mayor riqueza se encuentran gestantes pertenecientes a zonas urbanas $^{19,22}$.

A pesar las modificaciones en la maternidad y el embarazo en los últimos años ${ }^{15}$, se ha evidenciado en el presente estudio que las diferentes mujeres gestantes refirieron principalmente que no tenían la preferencia de quedar embarazadas en ese momento actual, siendo con mayor frecuencia en la región de la Costa $62.4 \%$, seguida de la Sierra $62.1 \%$ y en la Selva el $59.4 \%$.

En conclusión existen diferencias en las características sociodemográficas del embarazo según región natural en nuestro país, donde el nivel educativo, quintil de riqueza y la edad juegan un rol importante que contribuyen a conocer la evolución y las tendencias en el perfil de las gestantes.

Financiamiento: Autofinanciado.

Conflicto de intereses: Los autores declaran no tener algún conflicto de intereses.

\section{REFERENCIAS BIBLIOGRÁFICAS}

1. Borrell C, Cirera E, Ricart M, Pasarín MI, Salvador J. Social inequalities in perinatal mortality in a Southern European City. Eur J Epidemiol. 2003;18:5-13.

2. Agudelo-Londoño SM, González-Ortiz LD, Vélez-Álvarez GA, Gómez-Dávila J, Gómez-Arias RD. Análisis de las relaciones sociales durante el embarazo en mujeres con y sin morbilidad materna exᄀtrema. Medellín, 2011-2012. Rev. Gerenc. Polít. Salud. 2016; 15(30): 160-175. http://dx.doi.org/10.11144/ Javeriana.rgyps $15-30$.arsd

3. Zuleta J. La morbilidad materna extrema: una poาsibilidad para aprender de experiencias exitosas en el manejo de la complicacion obstétrica (Editorial). Rev Col Obs Gine. 2007; 58 (3): $180-1$.

4. Carbonell IC, López R, Alba Y, Gómez T, Smith L, Álvarez M, et al. Caracterización clínico-epidemio ᄀlógica de la morbilidad materna extremadamente grave. Santiago de Cuba 2009. Revista Cubana de Higiene y Epidemiología. 2009; 47 (3).

5. Álvarez-Carrasco R, Espinola-Sánchez M, AnguloMéndez F, Cortez-Carbonell L, Limay-Rios A. Perfil de incidencia de $\mathrm{VIH}$ en embarazadas atendidas en el Instituto Nacional Materno Perinatal de Lima, Perú. Ginecol Obstet Mex. 2018; 86(1):108-116. DOI: https://doi.org/10.24245/gom.v86i2.1900

6. Álvarez-Carrasco Ricardo, Espinola-Sánchez Marcos, Angulo-Mendez Fernando, Cortez-Carbonell Luís, Cabezudo-Reátegui Marina. Aplicación del algoritmo inverso para diagnóstico de sífilis gestacional en el Instituto Nacional Materno Perinatal, Perú. 2011-2017. Rev. chil. obstet. ginecol. 2018; 83( 4 ): 359-367. Disponible en: https:// scielo.conicyt.cl/scielo.php?script=sci_arttext\&pid=S071775262018000400359\&Ing=es. http://dx.doi.org/10.4067/ s0717-75262018000400359.

7. Cano-Serral Gemma, Rodríguez-Sanz Maica, Borrell Carme, Pérez María del Mar, Salvador Joaquín. Desigualdades socioeconómicas relacionadas con el cuidado y el control del embarazo. Gac Sanit. 2006; 20(1): 25-30. Disponible en: http:// scielo.isciii.es/scielo.php?script=sci_arttext\&pid=S021391112006000100005\&lng=es.

8. Almeida-Filho N, Kawachi I, Filho AP, Dachs JN. Research on health inequalities in Latin America and the Caribbean: bibliometric analysis (1971-2000) and descriptive content analysis (1971-1995). Am J Public Health 2003; 93:2037-43.

9. Salihu HM, Myers J, August EM. Pregnancy in the workplace. Occupational Medicine 2012; 62:88-97.

10. Organización Iberoamericana de Seguridad Social. Gestión de la protección de la mujer trabajadora en situación de embarazo y/o lactancia [Internet] 2015 [Consultado abril 30 
de 2019]. Disponible en: http://www.oiss.org/estrategia/IMG/ pdf/EOSyS-02.pdfL

11. Maroto-Navarro G, García-Calvente M, Mateo-Rodríguez I. El reto de la maternidad en España: Dificultades sociales y sanitarias. Gac Sanit. 2004; 18 Supl 2:13-23.

12. Braveman P, Tarimo E. Social inequalities in health within countries: not only an issue for affluent nations: Soc Sci Med 2002; 54:1621-35.

13. Marrero Santos ML, Román Hernández JJ, Salomón Avich $\mathrm{N}$. Estrés psicosocial laboral como factor de riesgo para las complicaciones de la gestación y el bajo peso al nacer. Rev Cubana Salud Pública [revista en Internet] 2013 [consultado el 30 de abril de 2019]. Disponible en: http://scielo.sld.cu/ scielo.php?pid=S0864-34662013000500006\&script=sci arttext

14. García Jordá Dailys, Díaz Bernal Zoe. Perspectiva antropológica y de género en el análisis de la atención al embarazo, parto y puerperio. Rev Cubana Salud Pública. 2010; 36(4): 330-336. Disponible en: http:// scielo.sld.cu/scielo.php?script=sci_arttext\&pid=S086434662010000400007\&lng=es.

15. Blázquez MI. Aproximación a la Antropología de la reproducción. Revista de Antropología Iberoamericana [serie en Internet]. 2005 [citado 30 abril 2019]. Disponible en: http:// www.aibr.org/antropologia/aibr/

16. Fuentes M. Salud reproductiva y salud integral de las mujeres. Reflexiones desde la práctica en Asistencia Sanitaria. En: Miqueo C, Tomás C, Tejero C, Barral MJ, Fernández T, Yago T, editores. Perspectiva de género en salud. Madrid: Minerva; 2001.

17. González P Rogelio. Salud materno-infantil en las Américas. Rev. chil. obstet. ginecol. [Internet]. 2010; 75( 6 ): 411-421. Disponible en: https://scielo.conicyt.cl/scielo.php?script=sci_
arttext\&pid=S0717-75262010000600011\&lng=es. http:// dx.doi.org/10.4067/S0717-75262010000600011.

18. Fondo de Población de las Naciones Unidas (UNFPA). El bono demográfico en las regiones del Perú. Lima: UNFPA; 2012.

19. Mendoza Walter, Subiría Gracia. El embarazo adolescente en el Perú: Situación actual e implicancias para las políticas públicas. Rev. perú. med. exp. salud pública. 2013; 30( 3 ): 471-479. Disponible en: http://www. scielo.org. pe/scielo.php?script=sci_arttext\&pid=S172646342013000300017\&lng=es.

20. Boyd C. La transformación de las mujeres rurales jóvenes en Perú. Análisis comparativo a partir de los censos nacionales (1961-2007). Documento de Trabajo N. ${ }^{\circ} 10$. Lima: Instituto de Estudios Peruanos, Nuevas Trenzas; 2013.

21. Escobar J, Saavedra J, Vakis R. ¿Está el piso parejo para los niños en el Perú? Medición y comprensión de la evolución de las oportunidades. Lima: Banco Mundial, GRADE; 2012.

22. Bayer AM, Tsui AO, Hindin MJ. Constrained choices: adolescents speak on sexuality in Peru. Cult Health Sex. 2010;12(7):771-82.

Correspondencia: Marcos Espinola Sánchez.

Dirección: C/ Lola Flores 4-17 Madrid.

Correo electrónico: marcosespinola.es@gmail.com

Teléfono: 913814091 